\title{
Energy and Round Time Estimation Method for Mobile Wireless Sensor Networks
}

\author{
NAJMA ISMAT*, REHAN QURESHI*, AND MUMTAZ-UL-IMAM* \\ RECEIVED ON 02.12.2016 ACCEPTED ON 21.02.2017
}

\begin{abstract}
Clustered WSN (Wireless Sensor Networks) is a hierarchical network structure that conserves energy by distributing the task of sensing and data transfer to destination among the non-CH (Cluster-Head) and CH (Cluster Head) node in a cluster. In clustered MWSN (Mobile Wireless Sensor Network), cluster maintenance to increase at a reception at the destination during communication operation is difficult due to the movement of $\mathrm{CHs}$ and non-CH nodes in and out of the cluster. To conserve energy and increased data transfer to the destination, it is necessary to find the duration after which sensor node's role should be changed from $\mathrm{CH}$ to non-CH and vice-versa. In this paper, we have proposed an energy independent round time scheme to identify the duration after which re-clustering procedure should be invoked for changing roles of sensor nodes as $\mathrm{CHs}$ and associated nodes to conserve energy and increased data delivery. This depends on the dissemination interval of the sensor nodes rather than sensor node's energy. We have also provided a complete analytical estimate of network energy consumption with energy consumed in every phase of a around.
\end{abstract}

Key Words: Mobile Wireless Sensor Network, Round Time, Cluster, Cluster-Head Rotation.

\section{INTRODUCTION}

$\mathrm{M}$ WSN is a collection of SN (Sensor Nodes) either deployed, implant, or worn by the nodes or entities that can move. Movement of SN makes efficient use of energy and performs better channel utilization and low latency [1,2]. The positive impact of node mobility in WSN is that it provides better network coverage by covering those are as that areas not covered with random deployment of SN [3]. MWSN application scenarios may include battlefield surveillance, disaster management, patient monitoring, animal behavioral studies, studying and monitoring seismic activities and underwater habitat, etc. Due to the nature of the application area and mobility of SN, clustering solutions available for static WSN are not applicable on MWSN.

Regardless of that the SN are stationary or mobile in a sensing field, the performance of WSN is measured in terms of energy consumption, reliability and network survival time [4-6]. The SN battery in general cannot be charged or placed once its operational and due to that the power management schemes and battery consumption methods determine the network life [7,8]. So, for increasing the network life, it is necessary to adopt methods that 
may conserve energy, for example, restricting network wide transmission of data and control packets, avoiding retransmission of lost packets by maintaining reliable connection between sender and receiver. Studies have shown that clustering methods are cost effective in terms of energy, scalability, fault tolerance, reduces latency, and decreases communication costs [9-13].

Communication in clustered networks takes place in rounds, which reduces the overall network energy usage and increases network life. Energy is conserved by changing the role of the $\mathrm{SN}$ as $\mathrm{CH}$ and as non- $\mathrm{CH}$ node in every round. This change in role of SN in every round distributes energy consumption among all the nodes. However, in many schemes round time is dependent on SN initial or residual battery power. So, if it is dependent on initial battery power it means that an increase in initial battery power increases the round duration. If round time is dependent on residual energy, then when CH battery passes the threshold limit, re-clustering procedure is invoked. The frequency of re-clustering increases with the decrease in CHs battery power and it further affects the network life. In any case, the round time is large at the beginning of network operation, forcing $\mathrm{CHs}$ to stay in their role for longer periods, which results in early depletion of their power. The overall result is non-uniform distribution of energy consumption that adversely affects the network's life time. In addition to the above mentioned drawbacks, we have also noticed that most of the energy estimation methods found in the literature for clustering protocols only consider the energy consumed in data transfer operation and it ignores the energy spent in exchange of control packets for network setup and management. This results in an inaccurate estimation of consumption of energy during network operation.

In this paper, we have presented new energy independent round time estimation method that determines when SN role should be changed from $\mathrm{CH}$ to non- $\mathrm{CH}$ node and conversely. The energy independent round time estimate shows that increase in SN initial battery power does not affect the cluster round time. An analytical estimate, for complete network energy consumption has also been given that includes energy consumed in exchange of data as well as control packets. The rest of this paper is organized as follows: Section 2 briefly discusses the previous work done in this area. Our proposed energy independent round time estimate is discussed in section 3. A mathematical expression for energy estimation is derived in section 4. Evaluation of our proposed work along with the discussion on the results are presented in section 5 and section 6 concludes the paper.

\section{RELATEDWORK}

Several round time estimation methods are proposed in research literature. These methods can be categorized as fixed, dynamic or adaptive estimation schemes. LEACH [14] a very popular distributed clustering solution for WSN and its round time is calculated from sensor nodes initial battery power, and the average energy consumed by $\mathrm{CH}$ and non-CH nodes in a round. Sensor nodes change its role from $\mathrm{CH}$ to non- $\mathrm{CH}$ role from $\mathrm{CH}$ to non$\mathrm{CH}$ node in every round is to distribute energy consumption among all nodes. In [15] the round time scheme is adopted from HEED [16]. The clustering process is divided into phases. Nodes are selected as $\mathrm{CH}$ in the in the first round of the setup phase. $\mathrm{CH}$ with residual energy within the threshold continues to be the $\mathrm{CH}$ in the next round. The re-clustering procedure is invoked when residual energy reaches the threshold energy. In [17] a hybrid cluster rotation strategy given. At the start of the network operation, SN have ample amount energy, so energy-driven rotation strategy is used which is dependent on the residual energy of the $\mathrm{CH}$. So, with network operation residual energy decreases and more energy are consumed in frequent $\mathrm{CH}$ election and re-construction of topology. When residual energy drops

Mehran University Research Journal of Engineering \& Technology, Volume 37, No. 1, January, 2018 [p-ISSN: 0254-7821, e-ISSN: 2413-7219] 
to a certain threshold, it switches to time-driven method which is a dependent node density, energy status and fixed when the number of rounds determined at the start of the network. LEACH-F $[18,19]$ is a dynamic, adaptive round time estimation method for fixed cluster networks has been proposed. In these schemes the duration of a round is determined from SN current energy. In each round elected $\mathrm{CH}$ sends current energy to the BS to calculate round time and broadcast it back to the SN. In [20] an adaptive scheme, where round time is calculated from the number of active nodes in the network. When the number of active nodes goes down to certain threshold number, round time is fixed to avoid frequent re-clustering.

Number of authors have proposed energy consumption estimation methods for distributed clustered networks. LEACH [14] protocol, which has been discussed widely by the research community lacks a complete energy estimation model. It estimates energy based on data transmitted in steady state phase and does not calculate the energy spent in exchange of control packets for cluster setup. For estimating the energy consumed for data transfer from SN to $\mathrm{CH}$ and from $\mathrm{CH}$ to sink in steadystate phase free space and two-ray ground propagation models are used. In [21] the authors have found the energy consumed in the setup phase of cluster formation and named it as overhead energy. The overhead energy includes energy consumed in sending and receiving control packets for cluster formation and energy consumed in node association with the $\mathrm{CH}$ and broadcast of TDMA schedules. WSN DEEC (Distributed EnergyEfficient Clustering) for heterogeneous [22] have calculated the network energy consumption of heterogeneous networks, but they have not considered the energy expenditure of the setup phase. DEEC gives the ideal estimates of the network life to compute the projected expenditure of energy by each node. In EERP (Energy Efficient Routing) and Clustering for MWSN [23], surplus energy of a $\mathrm{SN}$ is calculated. This estimation includes energy consumed in sensing, generating, transmitting, receiving and in standby mode. It does not consider the energy consumed in the exchanging control packets for the topology setup. In [24] energy usage estimation technique (LCEFCM) is presented, which computes the energy consumed in setup and steady-state phases of the Fuzzy C-Means clustering. It calculates the energy consumed by each $\mathrm{CH}$ and $\mathrm{CM}$ in setup and steady-state phase so around. In [25] energy estimation using the $\mathrm{M} / \mathrm{M} / 1$ queuing model has been proposed. They have estimated the energy consumption by the non$\mathrm{CH}$ nodes, $\mathrm{CH}$ nodes and the sink node in steady-state phase. The energy consumed by the node depends on number of receiving packets, reception energy, probability of node in idle state and the energy consumed in idle state.

\section{ENERGY INDEPENDENT ROUND TIME}

Clustering is a hierarchical topological scheme, where one complete communication operation takes place in around. Each round lasts for certain duration after which $\mathrm{CH}$ role changes to common sensing node. Due to this mechanism of change of roles in around, it is also known as $\mathrm{CH}$ rotation. Round time plays a very important role in the maintenance of topology as well as increasing the life time of the SNs [17].

The important factors that can affect the performance of clustered wireless sensor networks and its lifetime is its battery power, energy consumption model and round time. A round can be defined as the time interval during which network topology is created and data packet exchange takes place between $\mathrm{SN}$ and their $\mathrm{CH}$ and the data sink. The operations performed in a round, can be divided into two main phases, namely: setup phase and steady-state phase. Cluster creation takes place in setup phase and transmission of sensed data to its intended destinations takes place in steady state phase. Network

Mehran University Research Journal of Engineering \& Technology, Volume 37, No. 1, January, 2018 [p-ISSN: 0254-7821, e-ISSN: 2413-7219] 
topology is recreated at the start of every round by changing the roles of the $\mathrm{CH}$ to non- $\mathrm{CH}$ node and viceversa. The network lifetime also depends on round time, as it symbolizes the duration for which some SN are acting as $\mathrm{CH}$. The $\mathrm{SN}$ that acts as $\mathrm{CH}$ consumes more energy than common sensing node whose only job is to sense certain phenomena at regular intervals. $\mathrm{CH}$ not only performs the task of sensing but also acts as transit data hub for its cluster member to forward their sensed data to the sink. Shorter duration round means clustering procedure initiation frequency is high, which also consumes energy because of the frequent initiation of the setup phase. Longer duration round means early drainage of energy of those $\mathrm{SN}$ that are acting as $\mathrm{CHs}$ and thus compromising on network life.

The round time estimation methods can be categorized as fixed, dynamic or adaptive. Dynamic or adaptive schemes decide the duration of around from network statistics like remaining energy or number of alive nodes in a network. Dynamic or adaptive round time method given in [18-20] is applicable to networks where clustering is fixed and controlled by the base station. With dynamic, distributed clustering schemes adaptive round time is not applicable due its complexity of how to propagate network statistics and maintain same round duration among all the clusters. In fixed round time estimation scheme, base station decides the duration of a round and either provide priori information to $\mathrm{SN}$ at the time of deployment or propagate it in a network after deployment. Therefore, fixed round time estimation method is best suited for distributed clustered networks. In [14] authors proposed a ffixed round time estimation method for distributed clustering schemes. The drawback of this scheme is that it depends on SN initial battery power shown in Equation (1). The measure obtained from Equation (1) is approximately 20 seconds and with the assumption that SN initial energy $\left(E_{\text {initial }}\right)$ is 2 Joules $(J) t_{\text {frame }}$ is the total frame time and $9 \mathrm{~mJ}$ is the value obtained from number of frames per round. The main drawback of this scheme is increasing initial battery power increases round time, which means SN acting as $\mathrm{CH}$ are active for longer duration and thus consuming more energy and compromising on network life. Therefore, it is always good to provide a parameter that are perceptible for the realistic evaluation of the scheme.

$r_{t}=t_{\text {frame }} \times \frac{E_{\text {initial }}}{9 m J}$

In this work, we have proposed a new round time estimation method that is independent of SN initial battery power. It is the extension of the proposed VGDC protocol [25]. Our proposed estimation method does not consider any energy parameters, rather it depends on the size of the transmitted packet $m$ bits, data rate $\mathrm{R}$ as:

$r_{t}=t_{\text {setup }}+t_{\text {steady }}$

In the setup phase, clustered topological setup takes place, where clusters are formed before the transmission of data. In this phase four different types of control packets are exchanged between $\mathrm{CHs}$ and common SN. The time taken in setup phase as in Equation (3) consists of broadcasting of $\mathrm{CH}$ contention message $t_{c}$, transmission time of $\mathrm{CH}$ broadcast advertisements $t_{a d v}$, time taken for the transmission of join request message $t_{\text {join }}$, and time taken by TDMA schedule broadcast messages $t_{\text {tdma }}$ from CHs to its member nodes.

$t_{\text {setp }}=t_{c}+t_{a d v}+t_{\text {join }}+t_{\text {tdma }}$

The transmission of sensed data take place in steadystate phase and time taken $t_{\text {steady }}$ can be found from the time consume in transmission of m-bit message $t_{d f}$ over a communication channel that has a data rate of $R$ bits per second, $N_{c}$ number of nodes in a cluster and dissemination interval $\left(D_{\text {interval }}\right)$. 
$t_{\text {steady }}=N_{c} \times t_{d f}+\alpha \times D_{\text {interval }}$

The constant $\alpha$ can have a value based on the number of times data is disseminated by the SN during a round. It also signifies how long a node can act as cluster-head without draining its energy so not to compromise on network life.

Now, $t_{d f}$ is given as:

$\mathrm{t}_{\mathrm{df}}=\mathrm{m} / \mathrm{R}$

Also,

$$
\mathrm{N}_{\mathrm{c}}=\mathrm{N} / \mathrm{k}
$$

For the sake of simplicity assuming that $t_{c}, t_{a d v}, t_{\text {join }}$, and $t_{\text {tdma }}$ takes the same amount of time as the time taken by mbit message to transmit over $\mathrm{R}$ Mbps supported communication channel $t_{d f}$.

Now, solving Equation (3) and substituting the results and 4 in Equation (2), we have a CH round time $r_{t}$ :

$\mathrm{r}_{\mathrm{t}}=(\mathrm{N} / \mathrm{k}) \times(\mathrm{m} / \mathrm{R})+\alpha \times \mathrm{D}_{\text {interval }}+\mathrm{t}_{\mathrm{df}}+\mathrm{t}_{\mathrm{df}}+\mathrm{t}_{\mathrm{df}}$

So, cluster-head rotation or round time $\mathrm{t}_{\mathrm{r}}$ is given as:

$\mathrm{r}_{\mathrm{t}}=(\mathrm{N} / \mathrm{k}) \times(\mathrm{m} / \mathrm{R})+\alpha \times \mathrm{D}_{\text {interval }}+3 \times(\mathrm{m} / \mathrm{R})$

\section{ENERGY CONSUMPTION ESTIMATION}

Clustered networks support hierarchical communication where the entire sensing and transfer of the sensed data to its intended destination is performed in sessions known as rounds. The rounds are further divided into subsessions according to their working. In the first subsession network, topological setup takes place and in the second sub-session transfer of sensed data from SN to
$\mathrm{CH}$ and then to sink is performed. These two sub-sessions of a around are named: setup phase and steady-state phase respectively. Energy consumed in setup phase is mostly due to exchange of control information. This consumption of energy is significant, but often ignored because the number of control packets exchanged between nodes in setup phase is much less than the data packets once topology is created. The control information exchanged in setup phase is for the topology creation, and once topology is created, data transfer of sensed data can be initiated. Most of the energy consumed by $\mathrm{SN}$ is in a steady-state phase where some are acting as a $\mathrm{CHs}$ and some as common $\mathrm{SN}$ involved in sensing task only. The transfer of sensed data between SNs and their $\mathrm{CH}$ and from $\mathrm{CH}$ to a data sink takes place in this phase, so energy consumption much higher in this phase than in setup phase.

The energy estimation methods of MWSN protocols [2730] adopted the energy consumption model of [14], which only consider the energy consumed in the steady-state phase of a round. For an accurate calculation of utilized or consumed network energy, setup phase energy estimation is also necessary. In the setup phase cluster topology creation takes place for which control packets are exchanged between SNs. In this paper, we have proposed a complete network energy estimate that evaluates the energy spent on setup and steady-state phases of a round.

The energy consumed in each round can be found as:

$E_{r}=E_{\text {setup }}+E_{\text {steady }}$

In setup phase clusters are formed by selecting nodes as $\mathrm{CHs}$. The role of $\mathrm{CH}$ is to invite non-CH nodes to join its cluster by broadcasting cluster contention messages. After the completion of contention operation, sensor nodes elected as $\mathrm{CH}$, broadcasts $\mathrm{CH}$ advertisement 
messages to non-CH nodes. During setup phase, non$\mathrm{CH}$ nodes decide whether to become the part of the cluster by after receiving $\mathrm{CH}$ advertisements. They send join request to the $\mathrm{CH}$ if they decide to become cluster member. After receiving join request from the non- $\mathrm{CH}$ nodes, $\mathrm{CH}$ schedule data transfer operation among its members by issuing and broadcasting TDMA schedules. The energy consumed in setup phase is given as:

$E_{\text {setup }}=E_{\text {contention }}+E_{\text {CH-elec }}+E_{\text {tdma-sch }}$

$E_{\text {setup }}=E_{c \text {-Bradcast }}+E_{c \text {-Recv }}+E_{A D V-\text { Broadcast }}+E_{\text {ADV-Recv }}+E_{\text {join- }}$ Req $+E_{\text {Join-Recv }}+E_{\text {tdma-Broadcast }}+E_{\text {tdma-Recv }}$

The measures $E_{\text {contention, }}, E_{A D V-B r o a d c a s t}, E_{\text {join-Req }}$ and $E_{\text {tdma- }}$ Broadcast are given as:

$E_{\text {contention }}=\left(\left(N \times p_{\text {con }}\right) \times E_{c \text {-broadcast }}\right)+E_{c-\text { Recv }} \times\left(N \times\left(1-p_{\text {con }}\right)\right)$

$E_{\text {CH-elec }}=k x E_{A D V \text {-Broadcast }}$

$+(N-k) \times E_{A D V-R e c v}$

$+(N-k) \times E j_{\text {oin-Req }}$

$+E_{\text {Join-Recv }}$

$E_{\text {tdma-sch }}=k \times E_{\text {tdma-Broadcast }}+(N-k) \times E_{\text {tdma-Re cv }}$

Assuming that data and control messages are of size mbits, from [14], we have: Energy consumed in transmitting m-bit message is given as:

$E_{t x}(m, d)=E_{\text {elec }}(m)+E_{\text {amp }}(m, d)$

$E_{\text {tx }}(m, d)=E_{\text {elect }} \times m+E_{\text {efriss-amp }} \times d^{2}: d<d_{\text {crossover }}$

$E_{t x}(m, d)=E_{\text {elec }} \times m+E_{\text {efriss-amp }} \times d^{4}: d>=d_{\text {crossover }}$

Energy consumed in receiving m-bit message is:

$E_{r x}(m, d)=E_{\text {elec }} \times m$

Since, data and control messages are of equal size of mbits, so the energy consumed in transmission of control and data messages is the same as given in Equations $(14,15,17)$. The energy consumed in a broadcast operation like $E_{c \text {-Broadcast }}, E_{A D V \text {-Broadcast }}$ and $E_{\text {tdma-Broadcast }}$ use an Equation (16) whereas sending a join request may use Equation (15) or Equation (16) depending upon the distance of the receiving node. The energy consumed in receiving $\mathrm{CH}$ advertisements, join requests and TDMA schedules by sensor nodes irrespective of being a $\mathrm{CH}$ or not determined using Equation (17).

The operations performed in steady state phase are: sensing of phenomena, processing of data sensed from sensing phenomena, transmission of processed data from node to $\mathrm{CH}$ and from $\mathrm{CH}$ to the sink. It is possible that there some nodes that are not the part of any cluster, transmit their sensed data directly to the sink. The energy consumption in steady-state phase is given as:

$E_{\text {steady-state }}=E_{\text {sensing }}+E_{\text {processing }}+E_{t x-C H}+E_{r x-C H}+E_{t x-C H-B S}+E_{t x-\text { Node-BS }}$

The energy consumed in $E_{t x-C H}, E_{t x-C H-B S}, E_{t x-N o d e-B S}$ can be found from any of the Equations (17) or Equation (18), whereas $E_{r x-C H}$ can be obtained using Equation (19). Sensing energy $E_{\text {sensing }}$ and $E_{\text {processing }}$ is given by:

$E_{\text {sensing }}=P_{\text {sensing }} \times t_{\text {sensing }}$

$E_{\text {processing }}=P_{\text {processing }} \times t_{\text {processing }}$

So, the total energy consumed $E_{r}$ is found by substituting the values of Equations $(10,18)$ in Equation (9), we have:

$E_{r}=E_{c \text {-Broadcast }}+E_{c \text {-Recv }}+E_{\text {ADV-Broadcast }}+E_{\text {ADV-Recv }}+E_{\text {join-Req }}+$ $E_{\text {Join-Recv }}+E_{\text {tdma-Broadcast }}+E_{\text {tdma-Recv }}+E_{\text {sensing }}+E_{\text {processing }}+E_{t x-C H}+$ $E_{r x-C H}+E_{t x-C H-B S}+E_{t x-\text { Node-BS }}$

\section{EVALUATION OF THE PROPOSED SOLUTION}

For obtaining the experimental proof of our analytical work we have implemented our proposed work with VGDC protocol [26] and compared it with LEACH-M and grid clustering protocols. 
For simulations sensor nodes are randomly spread and moving with a speed of $0-5 \mathrm{~m} / \mathrm{sec}$ and $0-10 \mathrm{~m} / \mathrm{sec}$ in a sensing area of dimensions 150x150 and 200x200m. SN movement patterns are generated using a Bonn Motion Mobility pattern generator with different random seeds according to Gaussian-Markov model Symbols and their meanings used in this paper are shown in Table 1 and the summary of simulation parameters is shown in Table 2.

\subsection{Effects of Round Time}

To analyze the effects of round time, we have run simulations on VGDC protocol with different round times calculated from Equation (9) and evaluate data reliability at sink, network energy consumption and disconnection percentage of $\mathrm{CH}$ and its members. The round time values calculated from our work are 12, and 22 seconds when we

TABLE 1. SYMBOLS AND THEIR DEFINITION

\begin{tabular}{|c|c|}
\hline Symbol & Definition \\
\hline $\mathrm{N}$ & Number of sensor nodes \\
\hline pcon & contention probability \\
\hline $\mathrm{k}$ & Number of clusters \\
\hline $\mathrm{Nc}$ & Number of nodes in a cluster \\
\hline $\mathrm{m}$ & Packet size in bits \\
\hline Dinterval & Dissemination interval \\
\hline tdf & Time taken in transmission of m-bit message \\
\hline
\end{tabular}

TABLE 2. SIMULATION PARAMETERS

\begin{tabular}{|c|c|}
\hline Parameter & Values taken in simulation \\
\hline $\begin{array}{c}\text { Sensing Field } \\
\text { Dimensions }\end{array}$ & 150x150, 200x200m \\
\hline Node Density & 90,160 \\
\hline Data Sink Location & Center of the sensing field \\
\hline Initial Energy & $100 \mathrm{~J}$ \\
\hline Node Speed & $0-5$ and $0-10 \mathrm{~m} / \mathrm{sec}$ \\
\hline Eelec & $50 \mathrm{~nJ} / \mathrm{bit}$ \\
\hline Eamp & $10 \mathrm{pJ} / \mathrm{bit} / \mathrm{m} 2$ \\
\hline Etr & $0.0013 \mathrm{pJ} / \mathrm{bit} / \mathrm{m} 4$ \\
\hline Message Size $(\mathrm{m})$ & $400 \mathrm{bits}$ \\
\hline
\end{tabular}

assume that data is disseminated once and twice during a round respectively. For the sake of convenience, we named our protocol as VGDC-a when round time is 12 seconds and VGDC-b when round time is 22 seconds. The evaluated results have been compared with LEACH$\mathrm{M}$ and grid clustering methods for which the round time is fixed to 20 seconds as given by [14]. The reason of fixing this value for the two protocols is that it depends on SNs initial energy. Increase in initial energy of a SN increases round time, which means nodes acting as $\mathrm{CHs}$ continues their role for longer duration. They consume more energy than non-CH nodes, so there is a possibility of an early death of a network. We have also calculated the energy consumed in setup phase and the effect of different round times on setup phase energy consumption and discussed it detail in 5.2.

\subsubsection{Reliable Data Delivery}

To evaluate the performance of our proposed round time estimate, we have calculated the percentage of data packets delivered to the sink and named it as reliable data delivery. This includes the data packets send to the sink by CHs and nodes that are not associated with any cluster and exchanged data directly to the sink. Each sensor node in our analysis disseminates 49 data packets during the complete simulation run.

Fig. 1(a-b) shows that round time affects the successful data delivery to its final destination when nodes are moving with a speed of 0 and $5 \mathrm{~m} / \mathrm{sec}$ in sensing areas of 150x150 and 200x200m. At the sink data reception percentage increases with the decrease in round-time. The data reception percentage is approximately $88-93 \%$ for VGDC-a and 79-84\% for VGDC-b for different number of CHs. For the same number of CHs LEACH-M data reception percentage is in the range of $78-92 \%$. For grid clustering data reception at the sink is in between $74-90 \%$ for different cluster percentages. 
Similarly, for a nodal speed of 0 and $10 \mathrm{~m} / \mathrm{sec}$ in the same sensing fields as shown in Fig. 2(a-b), VGDC-a has the highest reception percentage than VGDC-b, LEACH-M and grid clustering for the same cluster percentages. This means that shorter round time offers stable clusters in MWSN where sensor nodes are moving with low and high speeds. It is possible that longer duration of the round may cause member nodes or $\mathrm{CH}$ to move out of their cluster and may result in increasing the distance between sensor nodes, $\mathrm{CH}$ and sink.

LEACH-M gives a high data reception percentage at the sink irrespective of the round time. The reason is that, it follows the principle of LEACH protocol, that makes broadcasting of control and data packets to ensure

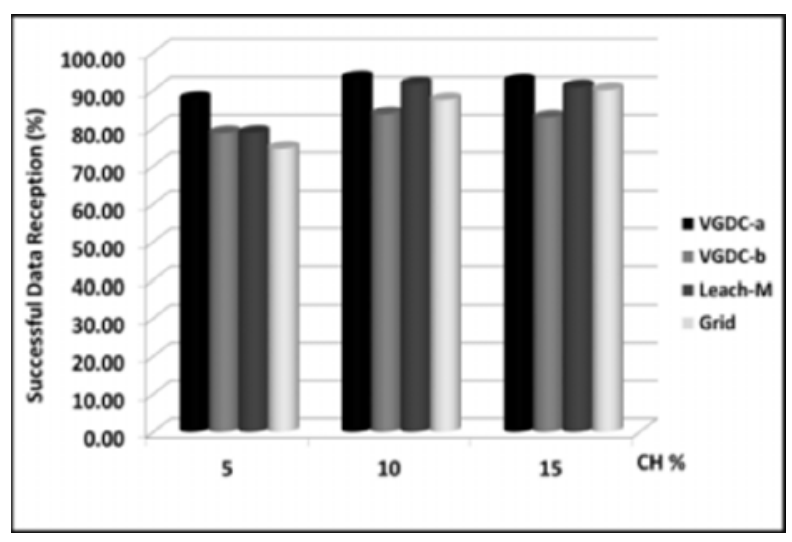

(a) $150 \times 150 m$

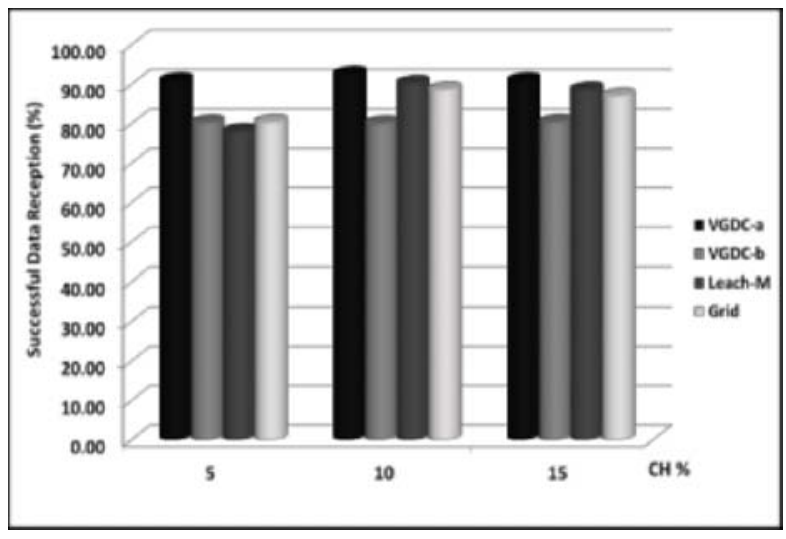

(b) $200 \times 200 \mathrm{~m}$

FIG. 1. DATA RECEPTION PERCENTAGE AT THE SINK WHEN NODE SPEED IS BETWEEN 0 AND 5 M/SEC delivery at the intended destination(s) at the cost of energy. Also, the number of packets send directly to sink by the nodes that have not been the part of any cluster is very high. Grid clustering shows higher data reception at the sink because each grid is considered as a cluster, therefore, complete cluster coverage is available throughout the sensing field.

\subsubsection{Cluster Maintenance}

By cluster maintenance, we mean that $\mathrm{CH}$ and SNs should maintain their association during a round irrespective of their movement in a sensing field. The nodes in a cluster is assumed to be associated with its $\mathrm{CH}$ if they are in the transmission range of each other. For this, we have

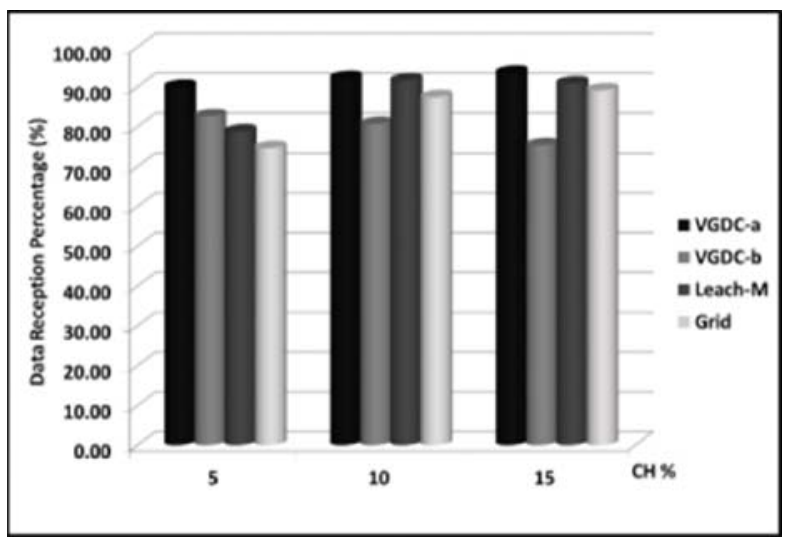

(a) $150 \times 150 m$

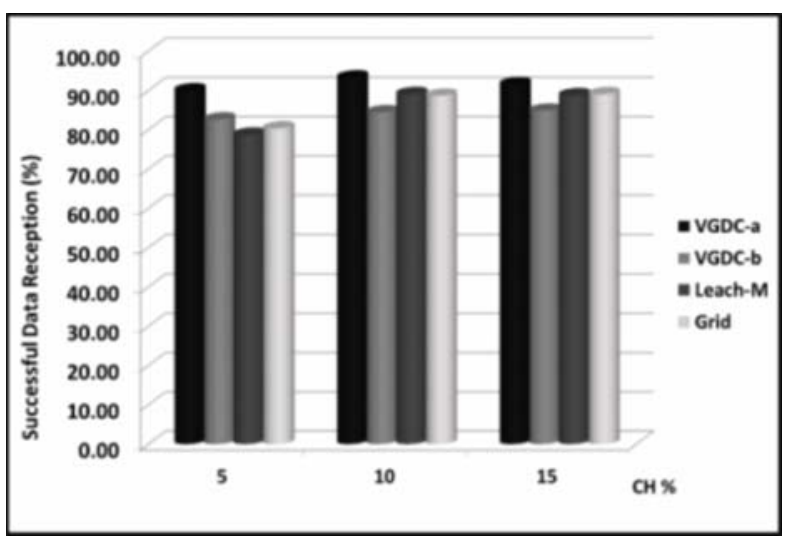

(b) $200 \times 200 m$

FIG. 2. DATA RECEPTION PERCENTAGE AT THE SINK WHEN NODE SPEED IS BETWEEN $O$ AND $10 \mathrm{M} / \mathrm{SEC}$

Mehran University Research Journal of Engineering \& Technology, Volume 37, No. 1, January, 2018 [p-ISSN: 0254-7821, e-ISSN: 2413-7219] 
calculated the disconnection percentage between them. It is obtained by taking the percentage of total number of times cluster-member or $\mathrm{CH}$ moves out from their cluster, with the distance between them is greater than their transmission range.

Fig. 3(a-b) and Fig. 4(a-b) shows the disconnection percentage between $\mathrm{CH}$ and its member nodes when they are moving with speeds between 0,5 and $10 \mathrm{~m} / \mathrm{sec}$ respectively. VGDC-a outperforms VGDC-b, LEACH-M and grid clustering for all speeds, sensing dimensions and $\mathrm{CH}$ percentages by providing better cluster maintenance strategy.

For node movement speed between 0 and $5 \mathrm{~m} / \mathrm{sec}$ as shown in Fig. 3(a-b) VGDC-a disconnection percentage

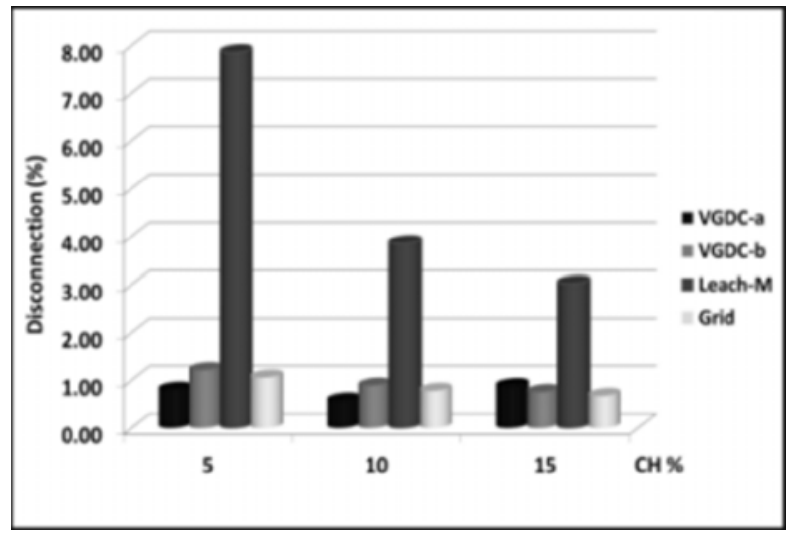

(a) $150 \times 150 m$

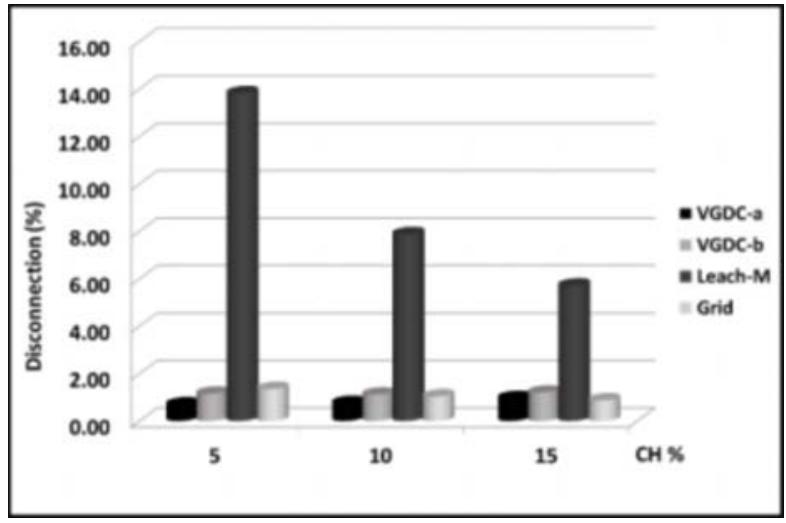

(b) $200 \times 200 m$

FIG. 3. CH-NODE DISCONNECTION WHEN NODE SPEED IS BETWEEN $O$ AND 5 M/SEC ranges between cluster members and their $\mathrm{CH}$ is in between $0.7-1.2 \%$ in $150 \times 150 \mathrm{~m}$ area and is between 0.7 $1.0 \%$ for $200 x 200 m$ area. Similarly, for LEACH-M this value is in between $3-8 \%$ in $150 \times 150 \mathrm{~m}$ and $5-14 \%$ for 200x200m sensing field size. The disconnection percentage value is increased for VGDC-b, but does not exceed $1.2 \%$ for all sensing field dimensions, which is still much less than LEACH-M. Fig. 4(a-b) compare the disconnection percentage of the three said protocols when nodal speed is 0 and $10 \mathrm{~m} / \mathrm{sec}$. Again, VGDC-a and VGDC-b outclass LEACH-M, and its disconnection percentage is maximum 7\% compared to 21\% of LEACH-M.

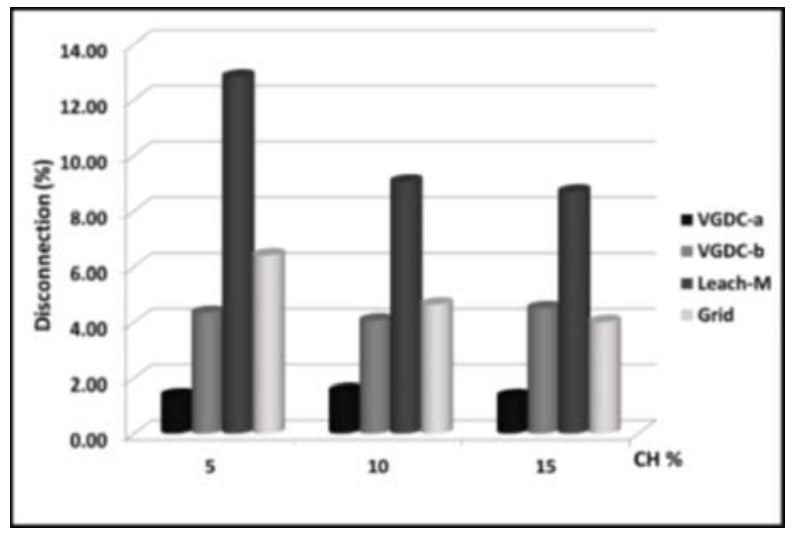

(a) $150 \times 150 m$

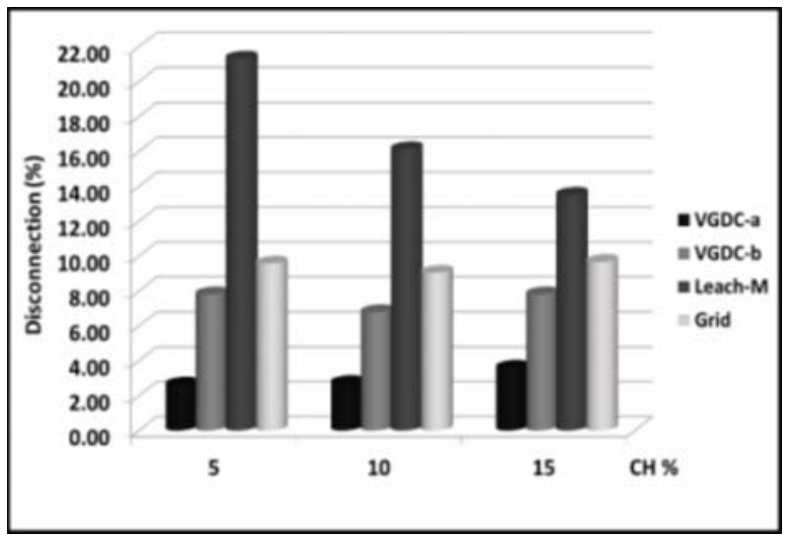

(b) $200 \times 200 m$

FIG. 4. CH-NODE DISCONNECTION WHEN NODE SPEED IS BETWEEN 0 AND $10 \mathrm{M} / \mathrm{SEC}$

Mehran University Research Journal of Engineering \& Technology, Volume 37, No. 1, January, 2018 [p-ISSN: 0254-7821, e-ISSN: 2413-7219] 
The disconnection percentages of VGDC and grid clustering protocols is approximately equivalent. Gird clustering protocol is slightly better than VGDC, in grid clustering because each grid is assumed as a cluster where as in VGDC one or more grids are within the cluster coverage depending on a gird size. In VGDC sensor nodes associates itself with the $\mathrm{CH}$ based on the cost metric which is the weighted sum of average received $\mathrm{CH}$ speed and distance from the SNs. The $\mathrm{CH}$ with the minimum or least cost is selected as its $\mathrm{CH}$ by the node and they associate themselves with it for the current round to send their data to the sink. Therefore, it is highly likely that if $\mathrm{CH}$ or its member relocates itself during a round it may still be within the communication range of each other.

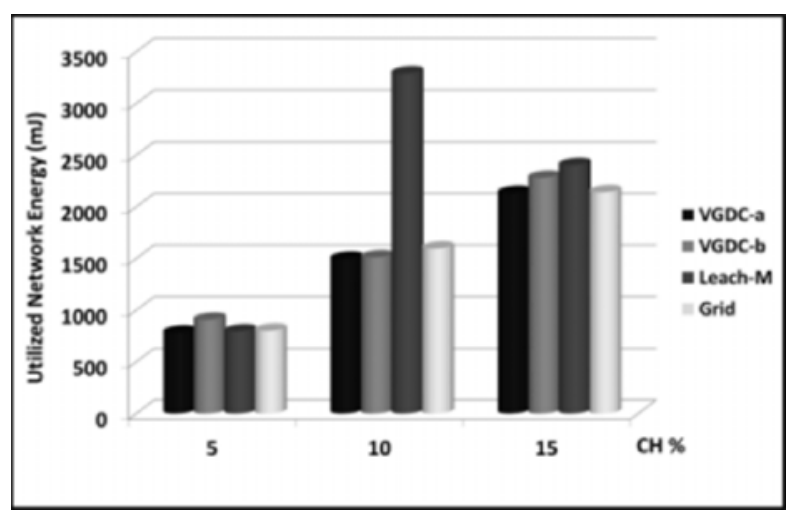

(a) $150 \times 150 m$

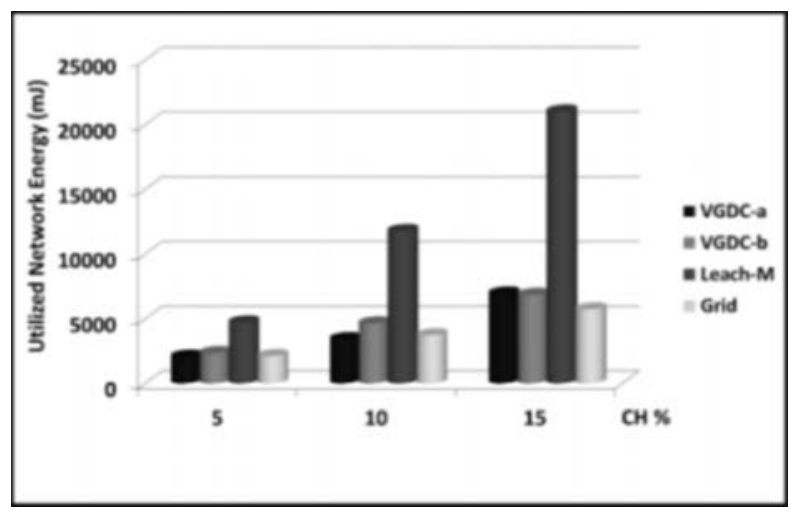

(b) $200 \times 200 m$

FIG. 5. UTILIZED NETWORK ENERGY WHEN NODE SPEED IS BETWEEN 0 AND $5 \mathrm{M} / \mathrm{SEC}$

\subsection{Network Energy Consumption}

For the analysis of energy usage, we have calculated the utilize network energy by noting down the energy spent in sensing, processing transmission and reception of control and data packets. In Figs. 5-6 we have compared the network energy consumed by the three said protocols with nodal speeds of $0-5 \mathrm{~m} / \mathrm{sec}$, and $0-10 \mathrm{~m} / \mathrm{sec}$ respectively.

Energy spent by VGDC-a and VGDC-b protocol is better than LEACH-M for all $\mathrm{CH}$ percentages, sensing field dimensions and speeds except for $5 \%$ clusters in 150x150m where it is slightly higher.

Due to location awareness, SNs in VGDC either $\mathrm{CH}$ or non-CH compute their current distance from data sink and transmit data with signal strength equivalent to carry

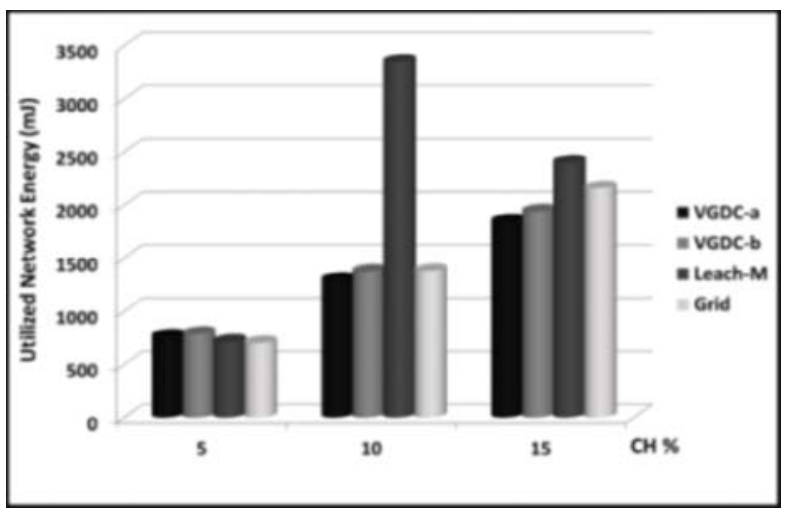

(a) $150 \times 150 m$

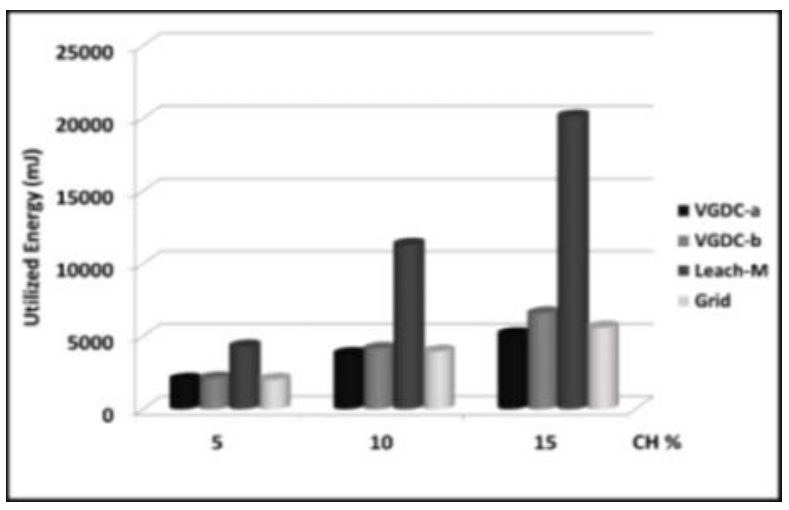

(b) $200 X 200 m$

FIG. 6. UTILIZED NETWORK ENERGY WHEN NODE SPEED IS BETWEEN 0 AND $10 \mathrm{M} / \mathrm{SEC}$

Mehran University Research Journal of Engineering \& Technology, Volume 37, No. 1, January, 2018 [p-ISSN: 0254-7821, e-ISSN: 2413-7219] 
data to that distance which conserves energy. In contrast to this, in LEACH-M for the transmission of control and data packet network wide broadcasts are done that result in better reception percentage of data packets at the sink but increased energy consumption.

The Grid clustering consumes more energy than VGDC-a protocol when nodes are moving with speed of 0,5 and $10 \mathrm{~m} / \mathrm{sec}$. This is because stable clusters are maintained with small round time and $\mathrm{CH}$-node association criteria of VGDC. Grid clustering shows slightly better results in contrast to VGDC-b when node speed is $10 \mathrm{~m} / \mathrm{sec}$ because when nodes are moving with high speed it is possible that nodes may move in and out of the grid during a round.

Also, in grid clustering each grid is a cluster so communication cost does not exceed the by setup phase is $1-6 \%$ for different cluster percentages in area of 150x150m shown in Fig. 6(a-b). The setup phase length of the sides of a grid. In VGDC the cluster radius comprises of one or more grid depending on grid size, so the communication cost may get increased.

\subsubsection{Setup Phase Energy Expenditure}

The effects of round time can further be analyzed with the energy consumed in the setup phase and the percentage of the utilized energy spent by the setup phase during communication. Smaller round time invokes cluster formation procedure more often and this causes an increase in the setup phase energy consumption. Fig. 7(a-b) shows the energy consumed in the setup phase VGDC-a and VGDC-b for sensing field sizes of 150x150 and 200x200m area with node mobility between 0,5 and $10 \mathrm{~m} / \mathrm{sec}$. Setup phase energy expenditure is high for VGDC-a and less for VGDC-b. For 5\% clusters setup phase energy expense is high, as compared to $15 \%$ clusters. This is because an increasing number of clusters increase the likelihood that sensor nodes are close to their nodes are moving with speed between 0,5 and $10 \mathrm{~m} / \mathrm{sec}$ respectively. The total energy consumed energy consumption percentage is in between $5-6 \%$ for VGDC-a when cluster percentage is 5 . The percentage of setup phase energy expense decreases with the increase in the number of clusters. The setup phase energy expenditure of utilized energy for the sensing field size of 200x200m shown in Fig. 8(a-b). This value ranges from 4-5\% low cluster percentage, but decreased with the increase in the number of clusters. Limiting the broadcast messages, transmission distances within the grid and within sensor node's transmission range, shows that VGDC protocol minimizes the energy consumed in the setup phase of a round. It also shows that invoking re-clustering procedure more often increased the energy usage, but at the same time increases the percentage of reliable transfer of data to its intended destination, by maintaining connectivity among $\mathrm{CHs}$ and associated SNs.

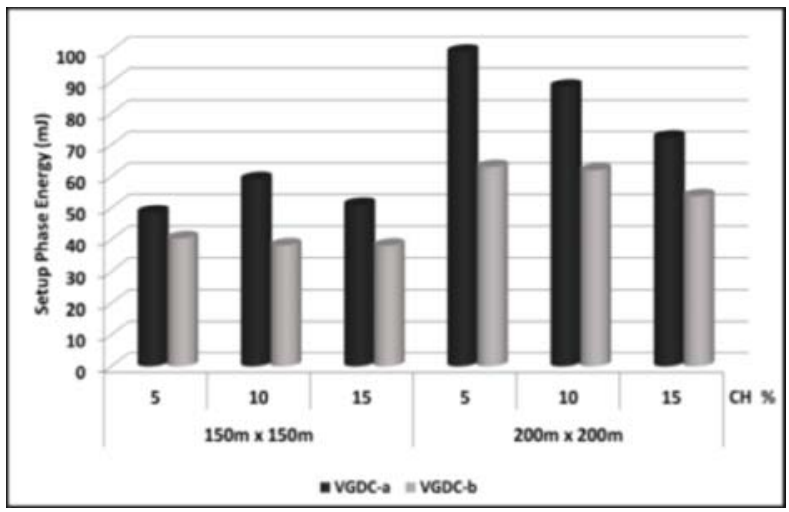

(a) NODE SPEED IS 0 AND $5 \mathrm{~m} / \mathrm{sec}$

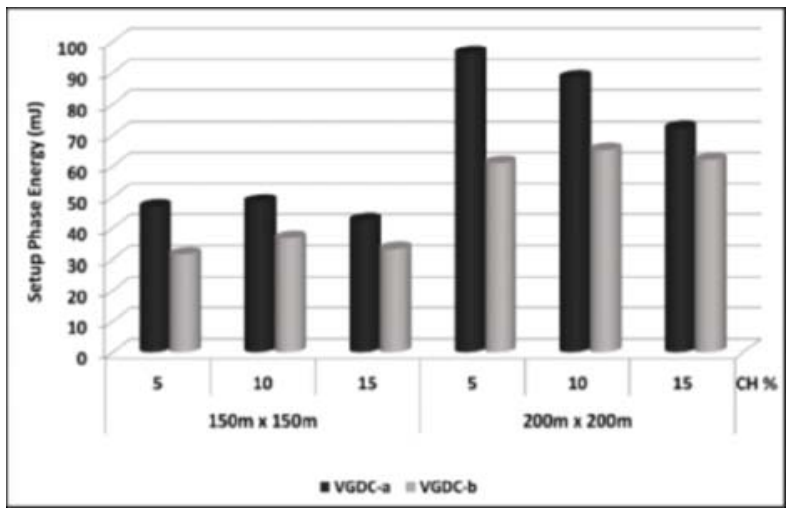

(b) NODE SPEED IS 0 AND $10 \mathrm{~m} / \mathrm{sec}$

FIG. 7. ENERGY CONSUMED IN SETUP PHASE

Mehran University Research Journal of Engineering \& Technology, Volume 37, No. 1, January, 2018 [p-ISSN: 0254-7821, e-ISSN: 2413-7219] 


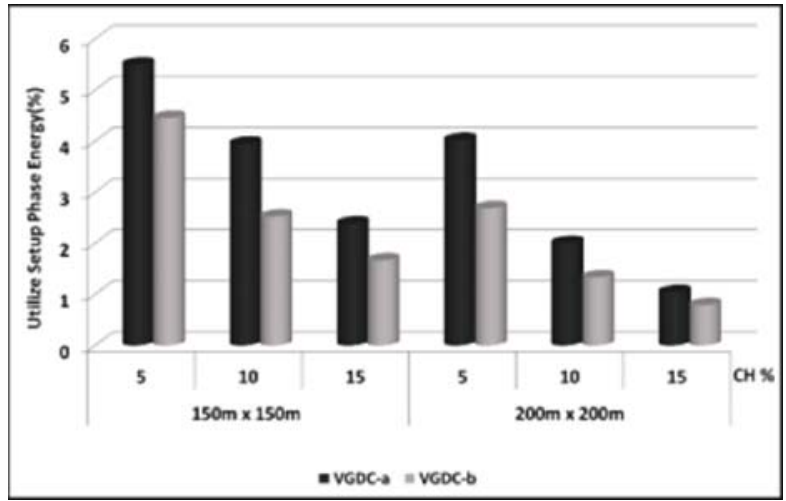

(a) NODE SPEED IS 0 AND $5 \mathrm{~m} / \mathrm{sec}$

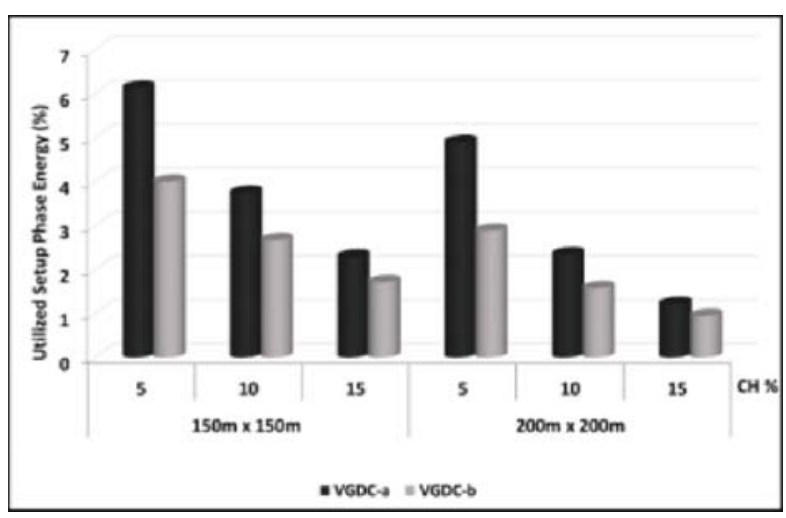

(b) NODE SPEED IS 0 AND $10 \mathrm{~m} / \mathrm{sec}$

FIG. 8. PERCENTAGE OF ENERGY UTILIZED IN SETUP PHASE

\section{CONCLUSION}

To conserve energy and increased data reliability, we have presented an energy independent round time estimation scheme for MWSN. Our proposed considers time taken by data and control packets to transmit to the destination, data dissemination interval and number of times data are disseminated in a round. This minimizes the possibility that round time expires while nodes are sending data to the destination. It also shows that smaller round time invokes clustering procedure more often but gives better data reliability in between $87-94 \%$. Shorter round time results in the creating more stable clusters by maintaining connectivity between $\mathrm{CH}$ and its member nodes. We have also given the analytical estimate of setup phase energy and proves that invoking clustering procedure more often means smaller round time consumes more energy in setup phase. It also shows that even with smaller round time the total percentage of energy consumed in setup phase is not more $6 \%$ from the total utilize network energy.

\section{ACKNOWLEDGEMENT}

The authors are grateful to the Sir Syed University of Engineering \& Technology, Karachi, Pakistan, for providing computing facilities in support of the research study. The university management has provided full support in the completion of the research.

\section{REFERENCES}

[1]

Kansal, A., Somasundara, A.A., Jea, D.D., Srivastava, M.B., and Estrin, D., "Intelligent Fluid Infrastructure for Embedded Networks", Proceedings of 2nd International Conference on Mobile Systems, Applications, and Services, pp. 111-124, Boston, USA, 2004.

[2] Santi, P., "Mobility Models for Next Generation Wireless Networks: Ad Hoc, Vehicular and Mesh Networks”, John Wiley \& Sons, February, 2012.

[3] Kumar, V., Dhok, S.B., Tripathi, R., and Tiwari, S., "A Review Study on Analytical Estimation of Optimal Number of Clusters in Wireless Sensor Networks", Transactions on Networks and Communications, Volume 2, No. 5, 2014.

[4] Sikander, G., Zafar, M.H., Raza, A., Babar, M.I., Mahmud, S.A., and Khan, G.M., "A Survey of ClusterBased Routing Schemes for Wireless Sensor Networks”, Smart Computing Review, Volume 3, No. 4, pp. 261-275, August, 2013.

[5] Ephremides, A., "Energy Concerns in Wireless Networks”, IEEE Wireless Communications, Volume 9, No. 4, pp. 48-59, August, 2002.

[6] Janani, E.S.V., and Kumar, P.G., "Energy Efficient Cluster Based Scheduling Scheme for Wireless Sensor Networks", The Scientific World Journal, Volume 2015, pp. 9, April, 2015. 
[7] Liu, B.-H., Pham, V.-T., and Nguyen, N.-T., “An Efficient Algorithm of Constructing Virtual Backbone Scheduling for Maximizing the Lifetime of Dual-Radio Wireless Sensor Networks", International Journal of Distributed Sensor Networks, Volume 2015, pp. 12, July, 2015.

[8] Al-Karaki, J.N., and Kamal, A.E., "Routing Techniques in Wireless Sensor Networks: A Survey”, IEEE Wireless Communications, Volume 11, No. 6, pp. 6-28, 2004.

Abbasi, A.A., and Younis, M., "A Survey on Clustering Algorithms for Wireless Sensor Networks", Computer Communications, Volume 30, Nos. 14-15, pp. 2826-2841, 2007.

[10] Siham, A., Driss, E. G., and Abdelilah, M., "Clustering Algorithms Based on Energy Efficiency in Wireless Sensor Networks: Survey”, ARPN Journal of Engineering \& Applied Sciences, Volume 8, No. 10, pp. 785-795, October, 2013.

[11] Lambrou, T.P., and Panayiotou, C.G., "A Survey on Routing Techniques Supporting Mobility in Sensor Networks”, IEEE 5th International Conference on Mobile Ad-Hoc and Sensor Networks, pp. 78-85, December, 2009.

[12] Akkaya, K., and Younis, M., "A Survey on Routing Protocols for Wireless Sensor Networks”, Ad hoc Networks, Volume 3, No. 3, pp. 325-349, 2005.

[13] Heinzelman, W.B., "Application-Specific Protocol Architectures for Wireless Networks”, Ph.D. Dissertation, Department of Electrical Engineering \& Computer Science, Massachusetts Institute of Technology, June, 2000.

[14] Mardini, W., Yassein, M. B., Khamayseh, Y., and Ghaleb, B.A., "Rotated Hybrid, Energy-Efficient and Distributed (r-Heed) Clustering Protocol in WSN", WSEAS Transactions on Communications, Volume 13, pp. 275-290, 2014.

[16] Younis, O., and Fahmy, S.,” Heed: A Hybrid, EnergyEfficient, Distributed Clustering Approach for Ad Hoc Sensor Networks”, IEEE Transactions on Mobile Computing, Volume 3, No. 4, pp. 366-379, OctoberDecember, 2004.
[16] Ma, G., and Tao, Z., “A Hybrid Energy and Time-Driven Cluster Head Rotation Strategy for Distributed Wireless Sensor Networks”, International Journal of Distributed Sensor Networks, pp. 13, January, 2013.

[17] Azim, A., and Islam, M.M., “A Dynamic Round-Time Based Fixed Low Energy Adaptive Clustering Hierarchy for Wireless Sensor Networks”, IEEE 9th Malaysia International Conference on Communications, pp. 1299-1302, Malaysia, 2009.

[18] Azim, A., and Islam, M.M., “A Relay Node Based Hybrid Low Energy Adaptive Clustering Hierarchy for Wireless Sensor Networks”, International Journal of Energy, Information and Communications, Volume 3, No. 3, pp. 41-54, August, 2012.

[19] Pal, V., Singh, G., and Yadav, R., "Network Adaptive Round-Time Clustering Algorithm for Wireless Sensor Networks”, International Conference on Advances in Computing, Communications and Informatics, August, 2013.

[20] Enam, R.N., Imam, M., and Qureshi, R., "Energy Consumption in Random Cluster Head Selection Phase of WSN", IACSIT Hong Kong Conferences, IACSIT Press, Edition, Volume 30, 2012.

[21] Qing, L., Zhu, Q., and Wang, M., “Design of a Distributed Energy-Efficient Clustering Algorithm for Heterogeneous Wireless Sensor Networks”, Computer Communications, Volume 29, No.12, pp. 2230-2237, 2006.

[22] Sara, G.S., Pari, R.K.N., and Sridharan, D., "Energy Efficient Clustering and Routing in Mobile Wireless Sensor Network", International Journal of Wireless \& Mobile Networks, Volume 2, No. 4, pp. 106-114, 2010.

[23] Raval, G., and Bhavsar, M., "Improving Energy Estimation Based Clustering with Energy Threshold for Wireless Sensor Networks”, International Journal of Computer Applications, Volume 112, No. 19, pp. 41-47, 2015.

[24] Rasouli, R., Ahmadi, M., and Ahmadvand, A., "Energy Consumption Estimation in Clustered Wireless Sensor Networks Using M/M/1 Queuing Model”, International Journal of Wireless \& Mobile Networks, Volume 5, No. 1, pp. 15-31, February, 2013. 
[25] Ismat, N., Qureshi, R., and Imam, M., "Efficient Clustering for Mobile Wireless Sensor Networks”, IEEE 17th International Multi Topic Conference, pp. 110-114, December, 2014.

[26] Kim, D-S. and Chung, Y.J., "Self-Organization Routing Protocol Supporting Mobile Nodes for Wireless Sensor Network", $1^{\text {st }}$ International Multi-Symposiums on Computer and Computational Sciences, Volume 2, pp. 622-626, 2006.

[27] Awwad, S., Ng, C., Noordin, N., and Rasid, M., "Cluster Based Routing Protocol for Mobile Nodes in Wireless Sensor Network,” Wireless Personal Communications, Volume 61, No. 2, pp. 251-281, 2011.
Kumar, G., Vinu Paul, M., Athithan, G., and Jacob, K., "Routing Protocol Enhancement for Handling Node Mobility in Wireless Sensor Network”, IEEE Region 10 Conference on TENCON, pp. 1-6, November, 2008.

[29] Awwad, S., Ng, C., Noordin, N., and Rasid, M., "Cluster Based Routing Protocol with Adaptive Scheduling for Mobility and Energy Awareness in Wireless Sensor Network”, Proceedings of the Asia Pacific Advanced Networks, pp. 57-65, 2010.

[30] Camp, T., Boleng, J., and Davies, V., "A Survey of Mobility Models for Ad Hoc Network Research”, Mobile Ad Hoc Networking Mobile Research, Trends and Applications, Volume 2, No. 5, pp. 483-502, August, 2002. 